\title{
Differential contributions of implicit and explicit learning mechanisms to various contextual cues in dual adaptation
}

\author{
Maria N. Ayala \& Denise Y. P. Henriques
}

The ability to switch between different tasks accurately and efficiently is an invaluable feature to a flexible and adaptive human motor system. This can be examined in dual adaptation paradigms where the motor system is challenged to perform under randomly switching, opposing perturbations. Typically, dual adaptation doesn't proceed unless each mapping is trained in association with a predictive cue. To investigate this, we first explored whether dual adaptation occurs under a variety of contextual cues including active follow-through movements, passive follow-through movements, active three-part lead-in movements, and static visual cues. In a final intervention, we provided our Instructed group with a compensatory strategy about the perturbations $\left(30^{\circ} \mathrm{CW} / \mathrm{CCW}\right.$ rotations) and their relationships to each context (static visual cues). This allowed us to explore the extent by which dual learning is supported by both implicit and explicit mechanisms, regardless of whether or not they elicited dual adaptation across all the various cues. To this end, following perturbed training, participants from all experiments were asked to either use or ignore the strategy as they reached without visual feedback. This Process Dissociation Procedure teased apart the implicit and explicit contributions to dual adaptation. We found that active movement cues, but not passive ones, elicited dual adaptation. Expectedly, static visual cues didn't elicit dual adaptation, but those in the Instruction group compensated by implementing aiming strategies. Critically, we found no implicit contributions in this Instruction group, but an effect of instruction, suggesting that explicit aiming strategies inhibit implicit mechanisms in dual adaptation. Thus, by implementing conscious strategies, dual adaptation can be easily facilitated even in cases where learning would not occur otherwise. 


\section{Introduction}

While it has always been imperative for humans to learn to manipulate new tools, with the

29 rise of modern technologies we are often required to operate and switch under multiple different

30 visuomotor mappings. This ability to switch between different tasks accurately and efficiently is

31 an invaluable feature to a flexible and adaptive human motor system. We can skillfully use a tool,

32 correct for any movement errors while we use it, and then anticipate the consequences of switching

33 to a completely different tool or environment. Often times we make large errors when first learning

34 to move under novel circumstances, but our motor systems quickly adapt, such that eventually,

35 and rather quickly, we produce smooth, accurate movements despite behavioural or environmental

36 constraints.

To study the ability of the motor system to switch between learned visuomotor maps,

38 variants of the same environment can be introduced serially (i.e. ABA paradigm) or concurrently

39 (i.e. dual adaptation paradigm). ABA paradigms probe how an internal model can be affected by

40 the subsequent learning of another, while dual adaptation paradigms have the advantage of

41 challenging the motor system to learn, acquire, and switch between multiple internal models,

42 mimicking its daily demands. Typically, when faced with unpredictable perturbations to

43 movement that vary from trial to trial, the motor system fails to adapt, leading to interference

44 (Brashers-Krug, Shadmehr, \& Bizzi, 1996; Caithness et al., 2004; Donchin, Francis, \& Shadmehr,

45 2003; Karniel \& Mussa-Ivaldi, 2002; Krakauer, Ghilardi, \& Ghez, 1999). However, adaptation to

46 two or more visuomotor perturbations (i.e. dual adaptation) can proceed when each visuomotor

47 map has been sufficiently cued during training, although what makes a cue sufficient is still

48 unclear. Usually, the relevance of the cue to the task and possibly the "intrinsic" or motor-based

49 nature of the cue appears to facilitate dual adaptation (e.g. (Ayala, 't Hart, \& Henriques, 2015; 
50 Howard, Wolpert, \& Franklin, 2013)), while cues that are more "extrinsic" or less pertinent to the

51 task lead to mixed findings (e.g. (Baldeo \& Henriques, 2013; Gupta \& Ashe, 2007; Hinder,

52 Woolley, Tresilian, Riek, \& Carson, 2008; Osu, Hirai, Yoshioka, \& Kawato, 2004)).

Adaptation to a single perturbation can be characterized by at least two qualitatively

54 different learning mechanisms: (1) an explicit, or conscious process which arises due to the

55 implementation of aiming strategies (Heuer \& Hegele, 2008; Taylor, Krakauer, \& Ivry, 2014), and

56 (2) an implicit process which is thought to function without awareness, reflect adaptation of

57 internal models in the cerebellum, and largely produce reach aftereffects (Heuer \& Hegele, 2008;

58 Taylor, Klemfuss, \& Ivry, 2010). Explicit and implicit contributions can also be elicited when

59 adapting to opposing visuomotor rotations for some contextual cues, such as when targets are

60 displayed in different workplaces (a cue that usually leads to dual adaptation rates similar to that

61 for single-rotation adaptation (Baldeo \& Henriques, 2013; Woolley, Tresilian, Carson, \& Riek,

62 2007)), and when the perturbations are large and blocked, as demonstrated by Schween and

63 colleagues (Schween, Taylor, \& Hegele, 2018). In one of their dual adaptation experiments,

64 Schween and colleagues showed that overlapping strategies led to interference, but when provided

65 with compensatory strategies, participants showed overall learning and reach aftereffects

66 (Schween et al., 2018). Still, it's unclear to what extent these underlying processes are engaged,

67 so here we break down the explicit and implicit contributions to dual adaptation across a diverse

68 set of contextual cues.

In this series of experiments, we explore the efficacy of various cues to dual adaptation,

70 including one involving an explicit aiming strategy. First, we looked at whether distinct active

71 movement sequences (i.e. follow-through movements) and more complex lead-in sequences can

72 facilitate adaptation to opposing visuomotor rotations. This has been similarly demonstrated under 
73 force-field paradigms (Howard, Ingram, Franklin, \& Wolpert, 2012; Howard, Wolpert, \&

74 Franklin, 2015), but it's unclear whether the active component of the contextual cue drove dual

75 adaptation, so in the following experiment we isolated the active movement component (i.e. the

76 self-generated follow-through component) and replaced it with a passive visual consequence that

77 mimicked that motion. We found that active, but not passive, movement sequences elicited dual

78 adaptation. We then equipped a separate Instruction group with a compensatory strategy about the

79 perturbations $\left(30^{\circ} \mathrm{CW}\right.$ and $30^{\circ} \mathrm{CCW}$ rotations) and their relationships to each context (visual

80 cues), and compared their performance to an uninstructed group with the same visual cues. Using

81 a Process Dissociation Procedure (PDP; adapted from (Werner et al., 2015), we teased apart the

82 implicit and explicit contributions to dual adaptation and found that visual cues didn't elicit dual

83 adaptation, but those in the Instruction group compensated by implementing aiming strategies.

84 Critically, we found no implicit contributions for those who were instructed, but an effect of 85 instruction, suggesting that explicit aiming strategies inhibit implicit mechanisms in dual

86 adaptation. Thus, by implementing conscious strategies, dual adaptation can be easily facilitated

87 even when learning would not occur otherwise.

\section{Method}

89 Participants

90 One-hundred and thirty-five participants ( 85 females, $20.40 \pm 3.23$ years old, mean \pm SD)

91 were recruited and assigned to participate in the following experiments and were granted a bonus

92 credit for either an undergraduate psychology or kinesiology course. All participants were right-

93 handed, had normal or corrected-to-normal vision, and were naïve to the purpose of the

94 experiment. Participants provided written consent in accordance with York University's Human 
95 Participant Review Committee. Two participants withdrew from the experiment due to discomfort

96 and were thus excluded from all analyses.

97 Apparatus

98 Participants sat on an adjustable chair facing a digitizing tablet (Wacom Intuos3, 12" x 12"

99 surface, resolution resampled by 1440 x 900 pixels at $60 \mathrm{~Hz}$ ) and a monitor (Dell Technologies,

100 22" P2217 LED screen) located approximately $55 \mathrm{~cm}$ from the tablet workspace. The tablet was

101 placed at waist level so that hand movements were made along the horizontal plane (Figure 1A).

102 To prevent participants from seeing their arm movements, an adjustable opaque shield was placed

103 above the tablet (Baldeo \& Henriques, 2013; Balitsky Thompson \& Henriques, 2010; Dionne \&

104 Henriques, 2008). All tasks required participants to reach to a target disc (1.5 cm in diameter) by

105 moving a digitizing pen that moved a hand-cursor $(1 \mathrm{~cm}$ in diameter $)$ in a 1:1 ratio. Some tasks

106 required a secondary reach from the target disc to a final target box $(3 \times 3 \mathrm{~cm}$, e.g. Figure $1 \mathrm{~B})$. The

107 target disc was radially-spaced and located at either $75^{\circ}, 90^{\circ}$, or $105^{\circ}$. In all experiments but one,

108 the target disc was flanked by a target box located $7.5 \mathrm{~cm}$ to its left or right, but never both at once.

109 All target discs began at a common starting point located $10.5 \mathrm{~cm}$ away from the home position.

110

111 


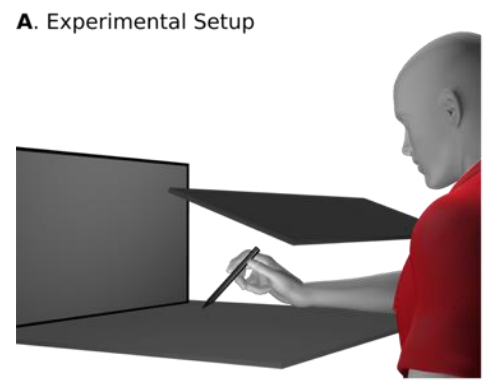

B. Active Movement Sequence

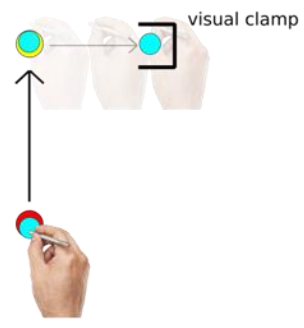

D. Task Display

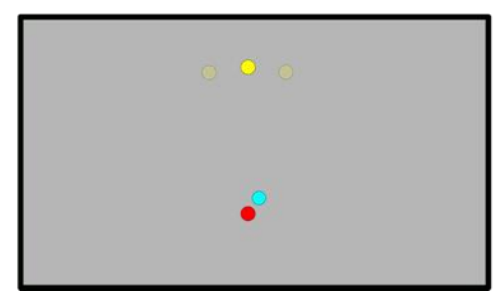

E. Static Visual Cue

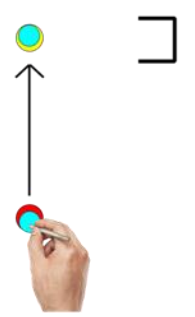

C. Passive Movement Consequence

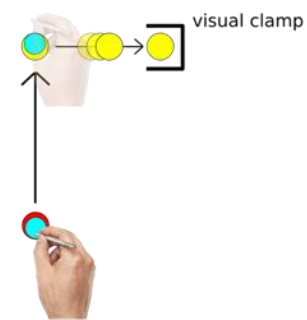

F. Active Lead-in Sequence

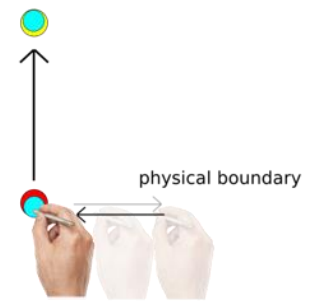

113 Figure 1. Experiment setup and task schematic. A. Participants reached along a horizontally114 placed digitizing tablet. An opaque shield occluded the view of their hand. Participants viewed 115 the motion of their hand-cursor on a monitor located at the end of the tablet. B. In the Active 116 Movement Sequence Experiment, participants reached to the yellow target disc, immediately 117 followed by a reach to the box target. CW-only and CCW-only control groups completed the 118 same task but only experienced a single perturbation. C. In the Passive Movement

119 Consequence Experiment, participants reached to the yellow target disc, stopped, and only 120 viewed the yellow target disc move to the target box. D. Participants saw targets discs located at 121 one of three positions. E. In the Static Visual Cue Experiment, participants only reached to the 122 yellow target disc. Those in the Instruction Experiment saw this same task but were instructed 123 a compensatory strategy. F. In the Active Lead-in Sequence Experiment, participants made an 124 unseen reach along a solid edge to the left or right, returned to the home position, and then to the 125 yellow target disc, completing a three-part sequence.

General Procedure whether distinct movement sequences facilitate dual adaptation by associating distinct movement sequences with opposing visuomotor rotations (Figure 1B). In the second experiment ('Passive

131 Movement Consequence Experiment"), we removed the active movement sequence and instead, 132 associated a distinct target consequence to each visuomotor rotation (Figure 1C). That is, instead 133 of moving to the target boxes, participants reached to the target discs, then simply viewed that 134 target disc directly move into the target box. In the third experiment ("Active Lead-in Sequence 
135 Experiment"), we implemented a more complex sequence of movements to cue the perturbations;

136 participants make an unseen leftward or rightward reach, then back to the home position, and

137 finally to the target disc, completing a three-part motion (Figure 1F). In the fourth experiment

138 ("Instruction Experiment"), participants were given instructions about the nature of the

139 perturbation and the relationship of the perturbations to the target boxes, although no movements

140 to the target boxes were required (Figure 1E). In the final experiment ("Static Visual Cue

141 Experiment"), like in the other experiments, left and right target boxes were coupled with

142 different perturbations, but no instructions were given and no secondary movement to the target

143 boxes occurred (i.e. purely visual cue; Figure 1E). Two groups, Single "CW-only" and "CCW-

144 only" served as control groups and learned only a single perturbation (thus, under only one context;

145 Figure 1B). Across all conditions, the presence of a left-flanking box was predictive of a $30^{\circ} \mathrm{CCW}$

146 rotation while the right-flanking box was predictive of a $30^{\circ} \mathrm{CW}$ rotation.

147 All participants started at the same home position and were told to make smooth and direct

148 reaches to the target disc. For trials involving visual feedback of the hand-cursor (also referred to

149 as "closed-loop trials"), participants simply reached towards the target disc. For trials involving

150 no visual cursor feedback (also referred to as "open-loop trials"), participants reached towards the

151 target disc with their unseen cursor, and remained stationary for $500 \mathrm{~ms}$ until the home position

152 appeared. In order to facilitate returning to the home position and proceed to the next trial during

153 open-loop trials, a line on the home position changed in orientation depending on the position of

154 the pen. A solid edge was also placed just below the home position to help participants find the

155 home position. Likewise, visual feedback of the hand-cursor appeared when participants were

156 within a $2 \mathrm{~cm}$ radius of the home position. 
For each experiment, participants completed pre-training, training, and post-training 158 sessions (Figure 2). For the $\mathbf{C W}$-only and CCW-only control conditions, participants completed 159 the task in approximately one hour (Figure 2A). For the rest of the experiments, participants 160 completed the task in approximately two hours (Figure 2B - C). With the exception of the 161 Instructions Experiment, participants were not informed about the presence or nature of the 162 rotation at the start of the experiments. After the experiment, participants answered a series of 163 questions to assess their awareness of the rotation and the experimental objectives. 


\section{A. Non-instructed single perturbation experiments}

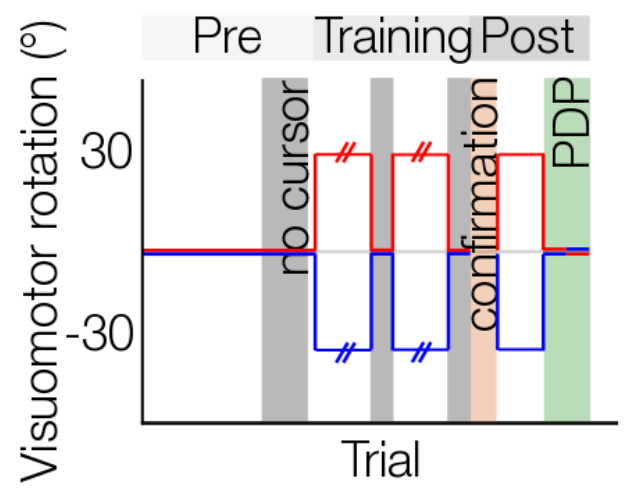

B. Non-instructed dual perturbation experiments

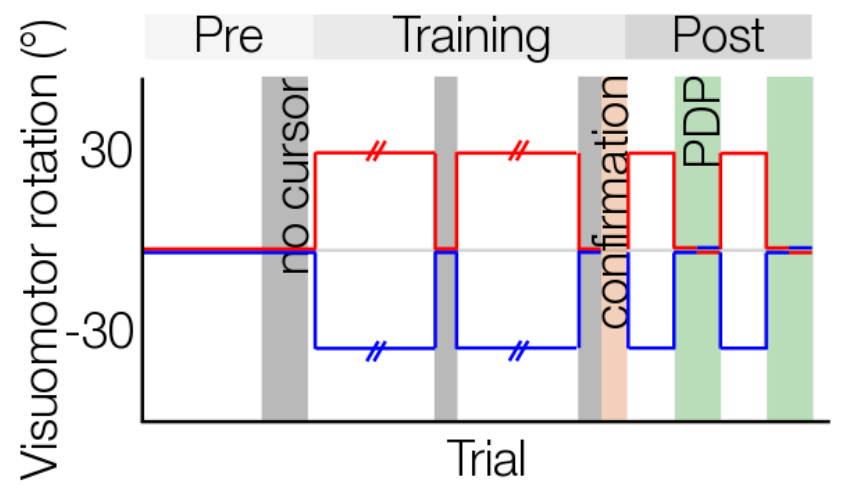

C. Instructed dual perturbation experiment

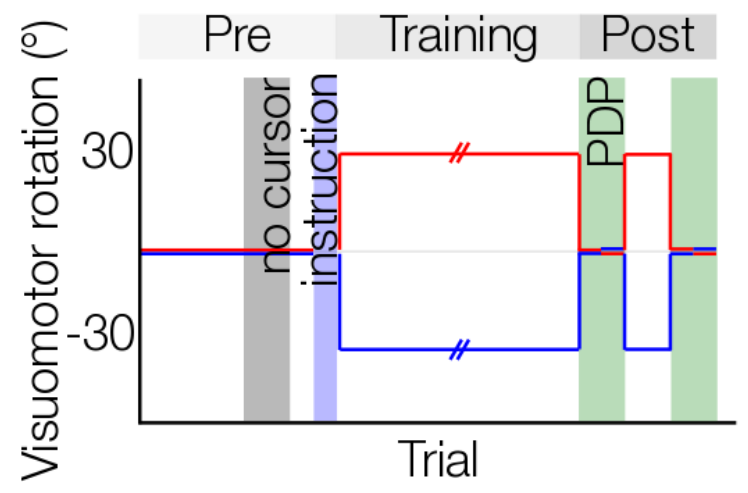

Figure 2. Perturbation schedule. A. Non-instructed single perturbation experiments. Two experimental groups follow this perturbation schedule: CW-only and CCW-only control groups. B. Non-instructed dual perturbation experiments. Four experiments fall into this perturbation schedule category: Active Movement Sequence Experiment, Passive Movement Consequence Experiment, Active Lead-in Sequence Experiment, and Static Visual Cue Experiment. B. Instructed Dual Perturbation experiment. Only the Instruction Experiment followed this

171 perturbation schedule. All PDP trials, shaded in green, were counterbalanced. 


\section{Active Movement Sequence Experiment}

Twenty-eight participants completed the Active Movement Sequence Experiment. In this

175 experiment, participants reached towards the target disc followed by a reach to either its LEFT or

176 RIGHT to a target box (Figure 1B). After acquiring the target disc, the hand-cursor was visually

177 clamped to only show the horizontal component of movement as they moved towards the target

178 box.

179 Pre-training (Baseline measures)

180 The aim of the pre-training session was to obtain baseline performance and familiarize

181 participants with the task. Participants reached towards the target disc 60 times with visual

182 feedback of the aligned cursor (veridical), followed by 24 reaches with no visual feedback of the

183 cursor (i.e. No-Cursor) in order to record baseline open-loop reach errors (Figure 2B, Pre-training).

184 Rotated Training (Adaptation)

185 The aim of reach training was to expose participants to a visuomotor rotation in order to 186 assess the learning rate and later, reach aftereffects. Participants reached 360 times with interleaved $18730^{\circ} \mathrm{CW}$ and $\mathrm{CCW}$ rotated cursors followed by 12 "Within-Training" No-Cursor reaches (Figure 188 2B, Training). This was repeated for a total of 720 rotated training trials. During training, each 189 rotation was associated with either a LEFT or RIGHT target box (Figure 1B), in order to determine 190 whether dual adaptation can occur when cued by different movement sequences. At the beginning 191 of every trial, participants saw both the initial and final targets. CW and CCW trials were presented 192 in a pseudo-randomized order such that participants encountered all three possible targets for each 193 cursor rotation before any target location was repeated.

194 Post-training (Analysis for awareness) 
196 move in the same direction as their hand, and that they needed to compensate and keep that

197 compensatory strategy in mind (Figure 2B, Confirmation, in orange), as they will later be prompted

198 by the screen to either use a "strategy" (Include-Strategy) or not use a "strategy" (Exclude-

199 Strategy). This Process Dissociation Procedure (PDP; adapted from Werner et al., 2015) allowed

200 us to make inferences about the participants' awareness, which in turn allowed us to tease apart

201 implicit and explicit contributions to dual adaptation. After Confirmation, participants reached

202 with both CW- and CCW-rotated cursors for 24 trials to preserve any dual adaptation. This was

203 followed by 12 Include-Strategy No-Cursor trials, then 12 Exclude-Strategy No-Cursor trials

204 (Figure 2B, Post-training). Finally, they again reached with both CW- and CCW-rotated cursors

205 for 24 trials, followed by another 12 Include-Strategy trials and 12 Exclude-Strategy trials. All

206 post-training tasks were counterbalanced.

207 Recall that we also collected No-Cursor reaches amidst training (Within-Training No-

208 Cursor trials). Previous dual adaptation work from our lab did not differentiate between Include-

209 Strategy or Exclude-Strategy No-Cursor trials; participants simply made open-loop reaches,

210 identical to our Within-Training No-Cursor trials here (Ayala et al., 2015; Ayala \& Henriques,

211 2018; Baldeo \& Henriques, 2013). Thus, to confirm that these previously found reach

212 aftereffects are indeed implicit in nature and to ensure that participants do not use any explicit

213 strategies without being reminded to do so, we will compare our Within-Training No-Cursor

214 trials (i.e. reach aftereffects after training) with Exclude-Strategy No-Cursor trials (i.e. reach

215 aftereffects after training and being told not to use any strategies).

\section{Single CW-only and CCW-only Control Experiments}


Twenty participants served as $\mathbf{C W}$ - or $\mathbf{C C W}$-only controls for all experiments. The task is

218 identical to the Active Movement Sequence Experiment, except participants in these groups 219 experienced either a $30^{\circ} \mathrm{CW}$ or $30^{\circ} \mathrm{CCW}$ rotation of the cursor during training. Participants 220 reached towards the target disc followed by a reach to either the left or right target box. Again, 221 after acquisition of the target disc, cursor movement was visually clamped to show only the

222 horizontal component of movement. The experiment began with a Pre-training session, consisting 223 of 60 aligned reaches followed by 12 No-Cursor reaches.

\section{Rotated Training (Adaptation)}

225 The aim of reach training was to expose participants to a visuomotor rotation in order to 226 assess the learning rate and later, reach aftereffects. During training, participants in the SINGLE 227 groups reached 180 times with a rotated cursor $\left(30^{\circ} \mathrm{CW}\right.$ or $\left.30^{\circ} \mathrm{CCW}\right)$ followed by 15 No-Cursor 228 reaches twice (Figure 2A, Training). This was repeated for a total of 360 rotated training trials. 229 Since participants in these groups only experienced a single perturbation, they only saw either a 230 left or a right target box across training. Trials were presented in a pseudo-randomized order such 231 that participants encountered all three possible targets before any target location was repeated.

232 Post-training (Awareness analysis)

233 As in the Active Movement Sequence Experiment, immediately after Training it was

234 confirmed with participants that the cursor would not move in the same direction as their hand and 235 that they needed to compensate (Figure 2A, Confirmation). They were told to follow screen 236 prompts that would state when (Include-Strategy) and when not to use a "strategy" (Exclude237 Strategy) to compensate for the odd movement of the cursor.

238 After Confirmation, participants reached with both CW- or CCW-rotated cursors for 24 239 trials to preserve any dual adaptation. This was followed by 12 Include-Strategy No-Cursor trials, 
240 then 12 Exclude-Strategy No-Cursor trials (Figure 2A, Post-training). All post-training tasks were

241 counterbalanced.

242 Passive Movement Consequence Experiment

243 Thirty participants completed the Passive Movement Consequence Experiment. Unlike

244 the Active Movement Sequence Experiment, participants simply had to reach to the initial target

245 and stay stationary until the initial target has made its complete migration into the final target

246 (Figure 1C). When participants failed to remain still, a high-pitched beep and a visual message

247 "Stay still!" signaled them to stop moving until all stimuli disappear.

248 Figure 2B shows the perturbation schedule for the Passive Movement Consequence

249 Experiment. Pre-training, training, and post-training followed the same protocol as in the Active

250 Movement Sequence Experiment (Figure 2B).

251 Active lead-in Sequence Experiment

252 Thirty-one participants completed the Active Lead-In Sequence Experiment. Instead of

253 making a secondary movement to the box target, participants made a three-part movement

254 sequence: first, an unseen initial reach to the left or right along a physical edge, followed by a

255 visually-clamped reach to the home position, then finally to the target disc (Figure 1F). Three

256 participants fell in the extreme outlier range and were excluded from analysis.

257 Figure 2B shows the perturbation schedule for the Active Lead-in Sequence

258 Experiment. Pre-training, training, and post-training followed the same protocol as in the Active

259 Movement Sequence Experiment (Figure 2B).

260 Instruction Experiment

261 Twelve participants completed the Instruction Experiment. Here, participants did not

262 reach towards the boxes or see any visual motions toward the boxes. Participants simply had to 
263 reach to the target disc and stay stationary (Figure 1E). When they failed to remain still, a high264 pitched beep and a visual message "Stay still!" signaled them to stop moving until all stimuli

265 disappear. Critically, participants were instructed about the nature of the perturbations and their 266 relationship to the static visual cues (i.e. boxes). They were explicit given a strategy on how to 267 counter the perturbations.

268 Figure 2C shows the perturbation schedule for the Instructions Experiment. During Pre269 training, participants reached towards the initial and final targets 60 times with visual feedback of 270 the aligned cursor, followed by 24 reaches with no visual feedback of the cursor (Figure 2C, Pre271 training). After Pre-training, participants were informed about the nature of the rotations and were 272 provided with a strategy to counteract the rotation so that they can move the cursor in a straight 273 path to the targets (Figure 2C, Instruction, shaded in blue). More specifically, they were instructed 274 to visualize the location of the home position as the center of a clock face. They were told that for 275 trials where they saw the box on the left side, reaching towards a number on the clock would result 276 in the cursor heading an hour behind, so they should aim an hour ahead to make a straight path to 277 the target. They were told that the opposite was true for trials where they saw the box on the right 278 side.

279 During training, participants reached 720 times with interleaved CW- and CCW-rotated 280 cursors (Figure 2C, Training). Post-training followed the same protocol as in the Active

281 Movement Sequence Experiment (Figure 2C, Post-training).

282 Static Visual Cue Experiment

283 Twelve participants completed the Static Visual Cue Experiment. This experimental

284 group served as controls for the Instruction Experiment; participants only saw the final target and 285 were not informed about the rotations, the coupling between the rotation and target box, nor were 
286 they given any compensatory strategy (Figure 1E). Participants reached to the target disc and

287 stayed stationary (and were otherwise alerted as described above in the Instruction Experiment

288 if they failed to do so). Figure 2B shows the perturbation schedule for the Static Visual Cue

289 Experiment. Pre-training, training, and post-training followed the same protocol as in the Active

290 Movement Sequence Experiment (Figure 2B).

291 Analysis

292 Cursor movement data was digitally smoothed using a first-order, low-pass Butterworth

293 filter with a frequency cut-off of $2.5 \mathrm{~Hz}$. Movement onset was fixed at $10 \%$ of peak velocity. The

294 experimental data was analyzed offline using R (preprocessing and analysis scripts can be found

295 in https://github.com/ayalamar/sequence), using kinematic error (operationalized as angular

296 deviation at maximum velocity) during training and No-Cursor trials. Angular error at maximum

297 velocity refers to the angular difference of the target and cursor at peak velocity relative to the

298 home position. For all our analyses involving blocks, we averaged across blocks of 3 trials in order

299 to include a movement to each target.

300 Visuomotor adaptation

301 Classic analysis of reach adaptation typically includes comparisons between single302 perturbation groups and dual-perturbation groups to compare rates of learning. However, as it has 303 been shown in the literature, dual adaptation rarely parallels the learning rate to that of single 304 adaptation, so we opted to report only dual learning statistics for brevity. For those who are 305 interested, omnibus tests are available on OSF and can be analyzed with scripts found in 306 (https://github.com/ayalamar/sequence).

307 The overall difference in reaching error between the first trial and the last block reflect the 308 extent of learning. Since CW and CCW rotations were not expected to elicit different rates of 
309 learning, we analyzed normalized errors, but we provide visualizations of signed errors to show

310 whether compensation occurs in the expected directions. A planned repeated-measures t-test (first

311 block vs. final block; one-sided) was used to show whether adaptation has occurred within each

312 experimental group. In addition, we compared performance from the beginning of rotation training

313 (substituted as 30 to reflect the size of the rotations) to the final block of experiencing the rotation.

314 We quantified this Percent Improvement (PI) below:

$$
\text { Percent Improvement }=\frac{\text { Initial block error }- \text { Final block error }}{30} \times 100
$$

316 Positive PIs indicate reduction in errors over time while negative PIs indicate worsening of errors

317 over time. PI was analyzed for every experimental group using a one-sample t-test, one-sided.

\section{Reach Aftereffects}

319 An alternative measure to test whether adaptation has occurred is the presence of reach

320 aftereffects during No-Cursor trials. Reach aftereffects refer to rotation-dependent, deviated

321 reaching in the absence of visual feedback of the cursor. Using the PDP (Werner et al., 2015),

322 reach aftereffects can be segmented to its explicit and implicit components. It is thought that

323 implicit learning is reflected in No-Cursor trials where participants are told not to implement any

324 strategy in their movements (i.e. Exclude-Strategy No-Cursor trials). To this end, we subtracted

325 any biases from the final block of No-Cursor trials following aligned training from the first

326 Exclude-Strategy No-Cursor trial, and determined whether this was significant from zero using a

327 one-sample t-test (one-sided) for all experimental groups. To assess whether participants were

328 using a compensatory strategy, we compared the first Include-Strategy No-Cursor trials and the

329 first Exclude-Strategy No-Cursor trials, after accounting for baseline biases, using a repeated-

330 measures t-test (one-sided) for all experimental groups. 
To confirm that Within-Training No-Cursor trials do not include explicit strategies, we also

332 compared whether errors during Within-Training No-Cursor trials differed from Exclude-Strategy

333 No-Cursor trials. Using a repeated measures t-test (one-sided), we found no differences in the

334 magnitude of reach aftereffects between the first Exclude-Strategy No-Cursor trial with the first

335 Within-Training No-Cursor trial, in both the Active Movement Sequence Experiment and

336 Active Lead-In Sequence Experiment.

337 The role of instruction

338 In this final set of analyses we set off to understand the role of explicit strategy in dual

339 learning by comparing the results between the Instruction Experiment (Instructed group) and the

340 Static Visual Cue Experiment (Uninstructed group). To confirm that participants in the

341 Instruction Experiment were able to implement the strategy, we compared their first block of

342 training to those from the Static Visual Cue Experiment using an Welch independent-measures 343 t-test.

344 First, to assess whether any implicit learning occurs following training, we ran a 2 X 2

345 Mixed Repeated-Measures ANOVA comparing reach aftereffects across Pre-training No-Cursor

346 tasks and Exclude-Strategy No-Cursor tasks between the Instructed and Non-instructed groups. A

347 significant effect would indicate the presence of reach aftereffects even when participants are

348 excluding strategy, suggesting the presence of an implicit learning mechanism.

349 Then, to examine the effect of instruction, we ran a 2 X 2 Mixed Repeated-Measures ANOVA 350 comparing across Exclude-Strategy No-Cursor tasks and Include-Strategy No-Cursor tasks

351 between the Instructed and Non-Instructed groups. If participants are aware and are using the

352 instructed strategy, we would see a significant difference between Include-Strategy No-Cursor

353 trials (in the expected directions) and Exclude-Strategy No-Cursor trials. Finally, this analysis will 
354 be followed by planned comparisons within each group (Instructed and Non-instructed groups) to

355 determine whether having instructions significantly affects the magnitude of reach aftereffects.

\section{Results}

To investigate the underlying mechanisms behind dual adaptation, we implemented

358 various contextual cues, and in one case, provided detailed instructions on how to counter the

359 perturbations given the contexts. In the Active Movement Sequence and Active Lead-in

360 Movement Sequence experiments, participants made two-part and three-part reaches that cued

361 the perturbations, respectively. In the Passive Movement Consequence, participants reached to a

362 target and saw the target move to a secondary target whose location cued the perturbation. In the

363 Instruction Experiment, participants were given a compensatory aiming strategy to dual adapt,

364 while those in the Static Visual Cue Experiment were left uninstructed. Across all experiments,

365 a leftward sequence (or visual location of the box) was indicative of a $30^{\circ} \mathrm{CCW}$ rotation, while a

366 rightward sequence (or visual location of the box) was indicative of a $30^{\circ} \mathrm{CW}$ rotation.

367 Participants in the Single CW-only and CCW-only control groups exhibited typical

368 visuomotor adaptation since they only encountered one perturbation, with large errors

369 approximately the size of the rotation at the beginning of training that gradually reduced to baseline

370 levels (Figure 3A), and rotation-dependent reach aftereffects (Figure 5A).

371 Visuomotor adaptation

372 Active movement sequence experiment

373 Participants made a two-part, active movement sequence involving a reach to the target disc

374 followed by a secondary, visually-clamped reach to the target box. Figure 3B (green) shows the

375 learning curves for Active Movement Sequence Experiment. We saw a small, significant

376 reduction in reaching errors across training $(\mathrm{t}(27)=2.45, \mathrm{p}=0.01)$. Distinct active sequences led 
377 to significantly improved performance for both rotations as seen in Figure 4 (green bar). On 378 average, participants significantly improved by a modest $16.25 \%$ across training $(\mathrm{t}(27)=1.86, \mathrm{p}=$ 379 0.04; Figure 4, green bar).

380 When we removed visual feedback of the cursor but kept the cues intact, we found significant 381 reach aftereffects in their expected directions $(\mathrm{t}(27)=5.13, \mathrm{p}<0.001$; Figure $5 \mathrm{~B}$, exclude strategy). 382 When asked to implement a compensatory strategy, participants do not change their reaching 383 behaviour $(\mathrm{t}(27)=-2.1942, \mathrm{p}>0.05$; Figure 5B, include strategy). These findings further support 384 that active movement sequences can facilitate dual adaptation, but this compensation is largely 385 implicit. This was further supported when we compared the size of reach aftereffects during 386 Within-Training No-Cursor trials and Exclude-Strategy No-Cursor trials and found no difference $387(\mathrm{t}(27)=-1.0626, \mathrm{p}>0.05)$. This suggests that participants do not implement any explicit strategies 388 during Within-Training No-Cursor trials, nor by the end of full dual training (when we directly 389 compare Exclude-Strategy and Include-Strategy reach aftereffects). 
A. CW- and CCW-only

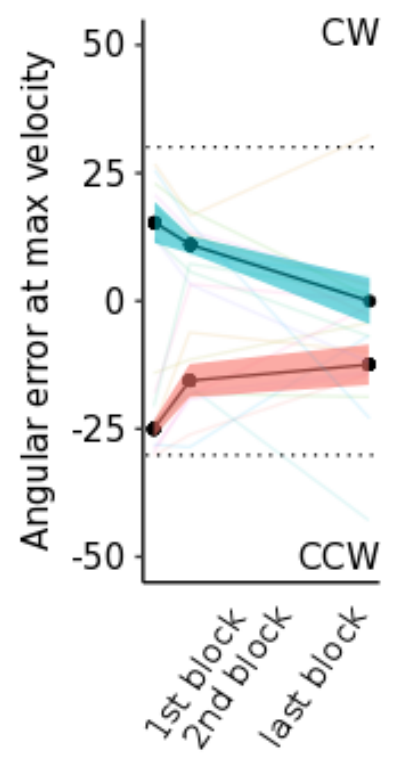

D. Active Lead-in

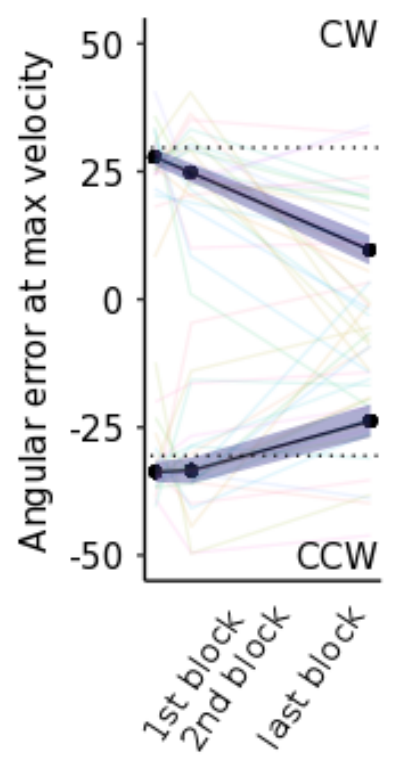

B. Active Movement

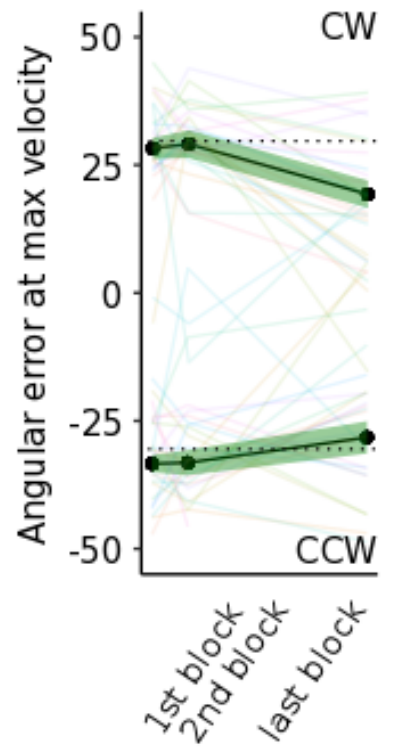

E. Explicit Instructions

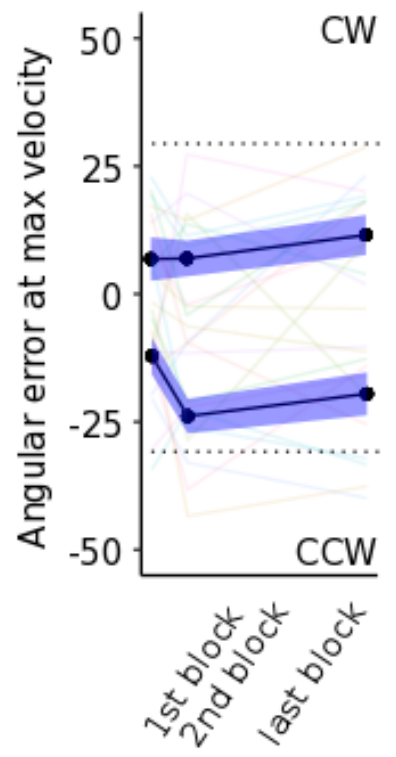

C. Passive Movement

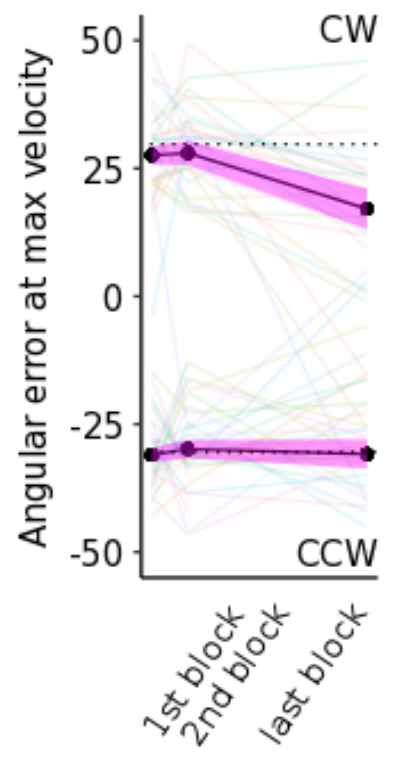

F. Static Visual Cue

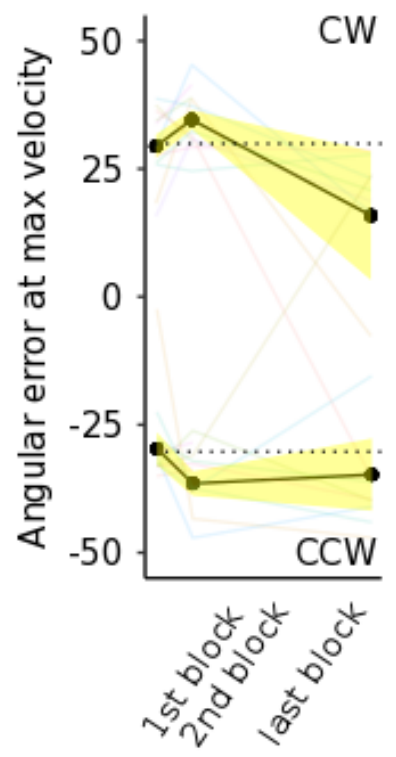

Figure 3. Visuomotor adaptation across training blocks. Colored translucent lines represent individual learning curves. A. Single CW- and CCW-only control groups. B. Active Movement Sequence Experiment. C. Passive Movement Consequence Experiment. D. Active Lead-in Sequence Experiment. E. Instructions Experiment. F. Static Visual Cue Experiment. Dashed 


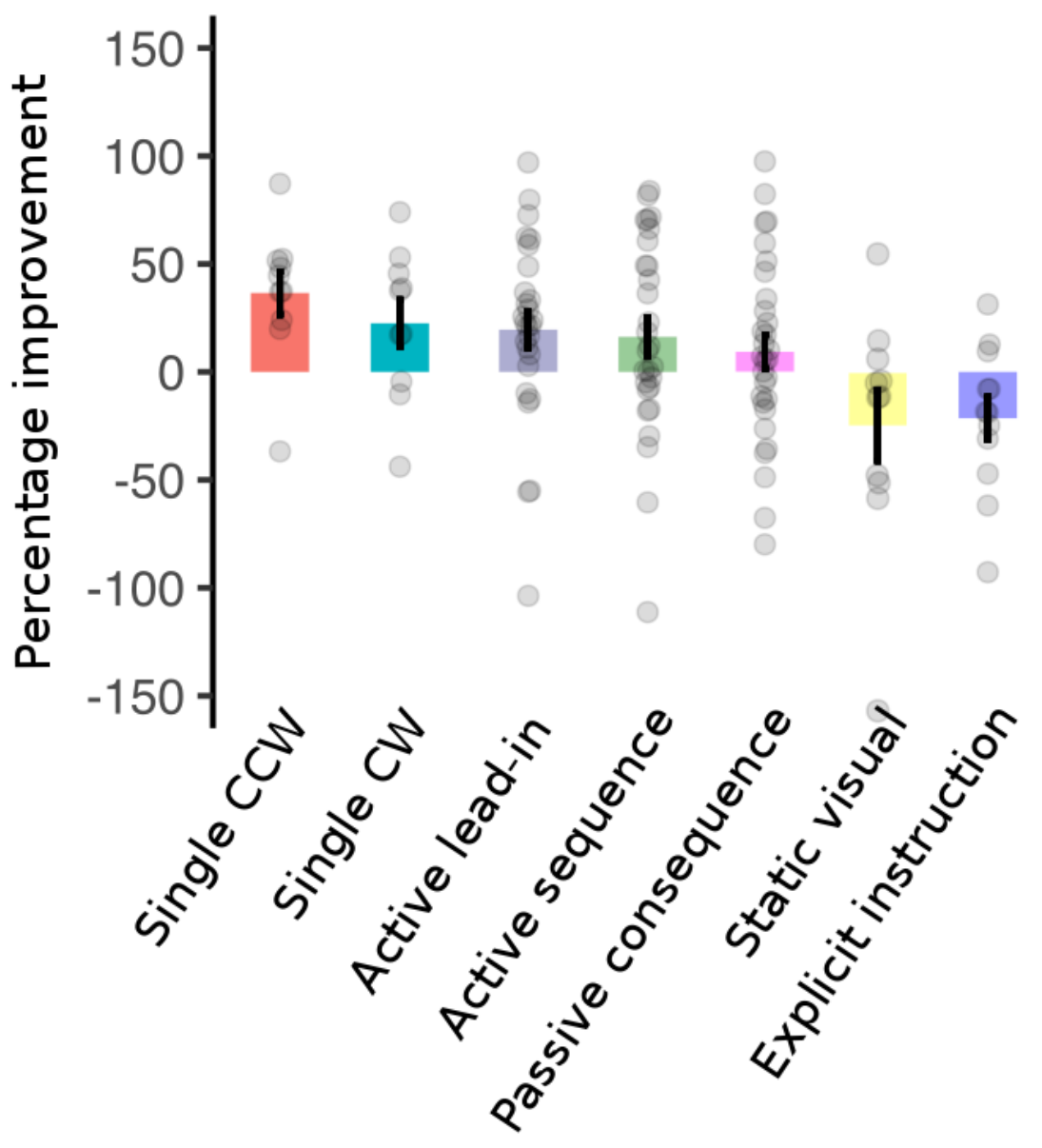

Figure 4. Percent improvement across training for all experiments. Grey dots represent 


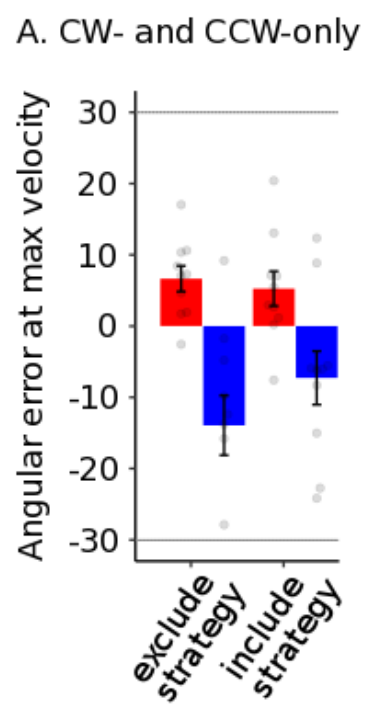

D. Active Lead-in

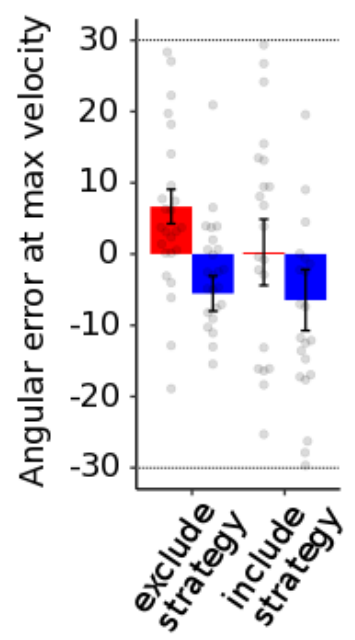

B. Active Movement

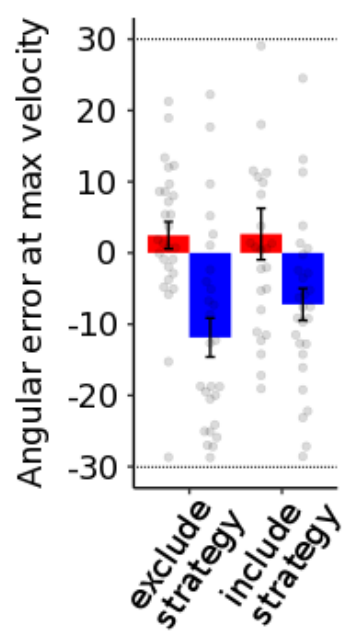

E. Explicit Instructions

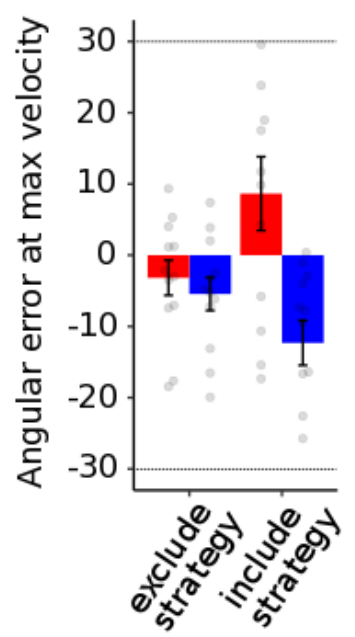

C. Passive Movement

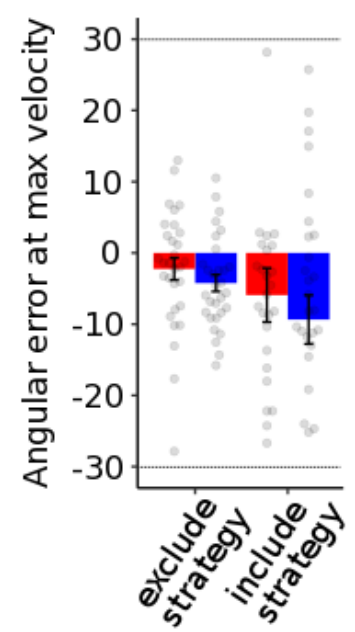

F. Static Visual Cue

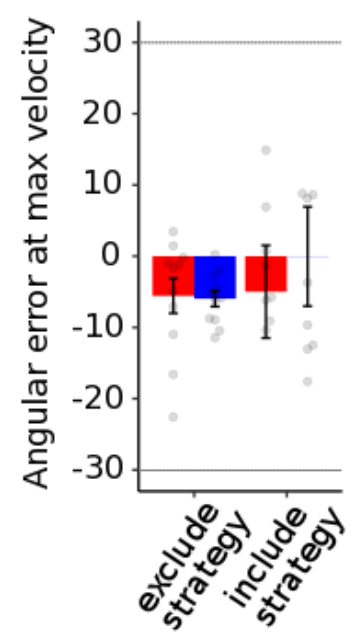

404 Figure 5. Reach aftereffects in post-training. Grey dots represent individual participant errors.

405 Red bars represent CW errors and blue bars represent CCW errors. Dashed lines represent

406 rotation magnitude experienced during training $\left(30^{\circ} \mathrm{CW}\right.$ and $\left.30^{\circ} \mathrm{CCW}\right)$. A. Single $\mathrm{CW}$ - and

407 CCW-only control groups. B. Active Movement Sequence Experiment. C. Passive Movement

408 Consequence Experiment. D. Active Lead-in Sequence Experiment. E. Instructions Experiment.

409 F. Static Visual Cue Experiment. Error bars represent SEM.

Passive movement consequence experiment

412 In contrast to the Active Movement Sequence Experiment, in the Passive Movement

413 Consequence Experiment participants were required to reach to the target disc with a CW- or 
414 CCW-rotated cursor, and then observe their target disc move towards the target box. Training

415 performance is illustrated in Figure 3C (pink). This cue was insufficient at facilitating dual

416 adaptation as evidenced by their large reaching errors that persist across training $(\mathrm{t}(29)=0.58, \mathrm{p}>$

417 0.05). This cue did not lead to any improvement over training $(\mathrm{t}(29)=1.18, \mathrm{p}>0.05$; Figure 4,

418 pink bar). Expectedly, reach aftereffects were not in the expected directions, and were not

419 significant from baseline $(\mathrm{t}(31)=1.34 \mathrm{df}=31, \mathrm{p}>0.05$; Figure 5C). Furthermore, participants did 420 not implement strategies in the correct directions $(\mathrm{t}(31)=-0.39, \mathrm{p}>0.05)$. Thus, merely viewing

421 the movement sequence does not elicit dual adaptation.

422 Active lead-in sequence experiments

423 To explore whether a more complex sequence of movements can elicit dual learning, we had 424 participants make a three-part movement sequence where the final portion of the reach was 425 perturbed but the preceding motions were predictive of that perturbation. In the Active Lead-in 426 Sequence Experiment, participants reached along an edge from the home position, back to the 427 home position, and finally, to the target disc. This active, three-part sequence was able to elicit 428 significant dual learning $(\mathrm{t}(27)=4.16, \mathrm{p}<0.001$; Figure 3D). On average, participants reduced 429 their errors by $19.57 \%$, and this improvement was significant from zero $(\mathrm{t}(26)=2.34, \mathrm{p}=0.01$; 430 Figure 4, purple bar).

431 Reach aftereffects for the Active Lead-in Sequence Experiment were in the expected 432 directions (Figure 5D). When asked to exclude any explicit strategy used, participants showed 433 significant reach aftereffects in their expected directions, suggesting the involvement of an implicit 434 mechanism $(\mathrm{t}(29)=2.23, \mathrm{p}=0.02)$. Conversely, when asked to implement an aiming strategy, 435 participants did not show any additional reach deviations in the expected directions as would be 436 expected if they had used a strategy $(\mathrm{t}(29)=-0.76, \mathrm{p}>0.05)$. 
To examine the explicit and implicit contributions to dual adaptation, we instructed a group

439 about the nature of the perturbations and how to compensate for them given the contextual cue

440 (Instruction Experiment) and compared their performance to a group who completed an identical

441 task but were not provided any instruction (Static Visual Cue Experiment). In the Static Visual

442 Cue Experiment, we eliminated any active or passive sequences; participants merely saw the box

443 targets but only reached towards the target discs. Expectedly, reaching errors did not reduce across

444 training $(\mathrm{t}(10)=-1.81, \mathrm{p}>0.05$; Figure 3F). On average, percent improvement was $-24.45 \%$ for

445 those who only received a static cue, which suggests that not only did participants fail to adapt,

446 they also performed worse over time (Figure 4, yellow bar).

447 Participants in the Instruction Experiment showed immediate benefits of strategy-use such 448 that they made smaller errors right at the beginning of training. On average, those with instructions 449 only had a $13.23^{\circ}$ error in the first block of training, which is significantly smaller than that of the 450 Static Visual Cue Experiment at $32.54^{\circ}(\mathrm{t}(20.80)=7.79, \mathrm{p}<0.001)$. This advantage was fleeting, 451 however, as participants in the Instruction Experiment do not further reduce their reaching errors 452 across training $(\mathrm{t}(11)=-1.78, \mathrm{p}>0.05)$, suggesting that instruction benefits are immediate and are 453 not moderated by more training (Figure 3E). Those in the Instruction Experiment did not show 454 any improvement over time $(\mathrm{t}(11)=-2.18, \mathrm{p}>0.05)$. Indeed, their average reaching error by the 455 end of training was only $18.41^{\circ}$, compensating for only about $38.64 \%$ of the rotation, implying 456 that the role of instruction is limited and exerts most of its effects in the early stages of learning 457 (Figure 3E).

458 Comparing No-Cursor trials across the Instruction Experiment (Figure 5E) and Static 459 Visual Cue Experiment (Figure 5F), we found no implicit learning such that Exclude-Strategy 
460 No-Cursor trials were no different from Pre-Training No-Cursor trials $(\mathrm{F}(1,11)=2.21, \mathrm{p}>0.05)$.

461 To probe the effect of instruction, we compared Exclude-Strategy and Include-Strategy No-Cursor 462 trials between Instruction and Static Visual Cue Experiments and found that having instructions 463 significantly elicits larger reach aftereffects in the expected directions $(\mathrm{F}(1,11)=10.72, \mathrm{p}<0.01)$. 464 Together, these findings suggest that when contextual cues convey no information, the motor 465 system can instead rely on an explicit mechanism to support adaptation.

To assess whether explicit processes are engaged within each experimental group, we 467 compared Include-Strategy No-Cursor trials with Exclude-Strategy No-Cursor trials for the 468 instructed and non-instructed groups separately. For the Instruction Experiment, we found that 469 explicit mechanisms are engaged such that Include-Strategy and Exclude-Strategy No-Cursor 470 reaches significantly differed $(\mathrm{t}(11)=2.61, \mathrm{p}=0.01$; Figure 5E). Expectedly, we did not find the 471 same for the Static Visual Cue Experiment $(\mathrm{t}(10)=-0.98, \mathrm{p}>0.05$; Figure 5F). Together these 472 findings suggest that when given an explicit compensatory strategy to dual adapt, the motor system 473 likely relies on explicit processes exclusively.

\section{Discussion}

475 To better understand what allows for dual learning, we investigated various contextual cues 476 including both actively-generated sequences and passive visual consequences. In a final 477 experiment, we explicitly provided participants with a compensatory strategy. Across all these 478 conditions, we teased apart the explicit and implicit mechanisms underlying dual adaptation by 479 separating the influence of strategy. We found that active movement sequences, but not passive 480 ones, facilitate dual adaptation. These simple active movement sequences were largely learned 481 without awareness, since we found implicit but not explicit contributions in their reach aftereffects. 482 Expectedly, static visual cues (a purely extrinsic cue), did not convey useful information to the 
483 CNS, leading to interference. However, when given instructions about the rotations and their

484 relationships to the static visual cues dual learning was able to proceed but only in the form explicit

485 learning, evident in the lack of implicit reach aftereffects.

486 Active but not passive cues facilitate dual learning

487 Previous work on dual adaptation has largely demonstrated that motor-based or "intrinsic" 488 cues tend to be more successful at reducing interference and facilitating learning (Ayala et al., 489 2015; Ayala \& Henriques, 2018; Baldeo \& Henriques, 2013; Galea \& Miall, 2006; Schween et 490 al., 2018; Wang \& Musseler, 2014; Woolley, de Rugy, Carson, \& Riek, 2011). We further 491 explored this idea by investigating whether self-generated movement sequence cues, which are 492 motor-based by nature, can facilitate dual adaptation. In both our Active Movement Sequence 493 and Active Lead-in Sequence Experiments we found significant dual adaptation as evidenced 494 by reduced reaching errors and significant reach aftereffects in the former experiment. Our 495 findings support the force-field work by Howard and colleagues, who found that distinct follow496 through motions can sufficiently cue the motor system to compensate for opposing force-field 497 perturbations (Howard et al., 2015). Still, it was unclear if movement sequences needed to be 498 self-generated in order to facilitate dual adaptation. To explore whether the active lead-in or 499 active follow-through was the key component in this contextual cue, we removed that active 500 component of the cue, as in the Passive Movement Consequence Experiment, and found no 501 reduction in reaching errors or any reach aftereffects in the expected directions. This suggests 502 that even dynamic visual cues-likely extrinsic like that used in the Static Visual Cue 503 Experiment, are insufficient even when it is temporally linked as a consequence of the perturbed 504 reach. 
Research by Howard and colleagues also found that lead-in motions can be coupled with opposing force-fields to produce significant reach aftereffects (Howard et al., 2012). They found

507 that in their most effective dwell condition that included a lead-in motion, participants were able

508 to compensate up to $75 \%$ of the necessary force to overcome the perturbation. Here, we found

509 that participants compensated for less than half the size of the rotation in the Active Lead-in

510 Sequence Experiment. There are a few factors that may account for this. One key difference is

511 the complexity of our lead-in cue-participants were asked to make a three-part motion in which

512 the final reach was perturbed. So, not only did participants in the Active Lead-in Sequence

513 Experiment have to access the past motion as a cue (second lead-in motion to the home

514 position), they also had to go one step back to access the motion cue prior to that (first lead-in

515 motion from the home position). Thus, one possibility is that cues that take longer to execute

516 hampers its efficacy. Indeed, Howard and colleagues have found that a complex combination of

517 lead-in and follow-through motion cues elicited dual adaptation to opposing force-fields, but

518 only after intensive training across 5 days (Howard et al., 2015). Additionally, in a previous

519 study of ours, we saw that doubling the training (from 360 to 720 trials) did not sufficiently

520 induce greater improvement nor larger reach aftereffects (Ayala et al., 2015), so it's possible that

521 this is the maximum level of dual adaptation for this specific cue. Thus, future work should

522 investigate if, and to what extent, longer sequences elicit dual learning. An alternative and less

523 prevalent account might be that certain cues are more effective at facilitating dual adaptation to

524 more dynamic tasks (i.e. force-field perturbations), and less to visuomotor rotations like we saw

525 in our present experiments. Together, these may account for a proportion of why we saw a

526 smaller mean level of compensation compared to previous force-field work implementing similar

527 cues. 
Interestingly, knowledge about the perturbations alone does not seem to dictate whether dual

529 adaptation will occur. While not reflected in larger Include-Strategy reach aftereffects, post-

530 experimental interviews revealed that a large proportion of those in the Active Lead-in

531 Sequence Experiment were aware of the perturbations. In fact, in the Active Lead-in Sequence

532 Experiment, nearly $42 \%$ (13 out of 31) of participants were able to explain the nature of the

533 perturbations and their relationships with the cues, compared to 8 out of 28 participants (28.57\%)

534 in the Active Movement Sequence Experiment. This didn't seem to lead to any advantage as

535 both active experiments achieved a similar level of percentage improvement. For the

536 experiments where dual adaptation did not occur, only one participant in the Static Visual Cue

537 Experiment and an intriguing 8 out of 30 (26.67\%) participants in the Passive Movement

538 Consequences Experiment were able to explain the nature of the perturbations and their

539 relationships to the cues. Thus, it seems that even with a sizeable portion of participants having

540 knowledge about the nature of the perturbation, we still do not see significant strategy

541 application. Possibly, insufficient cues might require the implementation of explicit aiming

542 strategies to be successful.

\section{Dual reach aftereffects are implicit by default}

544 In our previous dual adaptation experiments, we collected reach aftereffects during No-

545 Cursor trials without prompting participants that the rotations have been removed and thus,

546 without any reminder to ignore any strategy they may have been implementing during training

547 (Ayala et al., 2015; Ayala \& Henriques, 2018; Baldeo \& Henriques, 2013). To validate our

548 previous findings, here we confirm that Within-Training reach aftereffects (i.e. following

549 training but without prompt to exclude strategy) do not differ in magnitude compared to

550 Exclude-Strategy reach aftereffects (i.e. following training but with instructions to exclude any 
552 Experiment. This suggests that reach aftereffects from our previous experiments are likely due

553 to implicit mechanisms and excludes any conscious aiming strategies. Thus, when reaching

554 without visual feedback of the cursor, regardless of whether or not they are asked to, participants

555 tend to disregard any strategies by default. Still, it's possible that this applies only for moderately

556 effective cues, while stronger, more predictive cues such as workspace location (Baldeo \&

557 Henriques, 2013; Schween et al., 2018) are able to recruit not only implicit, but also explicit

558 mechanisms.

\section{Insufficient cues can be salvaged by aiming strategies}

As expected, static visual cues did not show any predictive strength, so in addition we

561 provided participants in the Instruction Experiment with aiming strategies that depended on

562 that context. This implementation led to significant learning which suggests that when cues are

563 insufficient (e.g. static visual cues), the CNS can tap into explicit mechanisms to support dual

564 learning. This purely explicit learning, however, comes at a cost. We saw that those in the

565 Instruction Experiment were able to immediately compensate by implementing the aiming

566 strategy, but they failed to improve, only retaining a small portion of the aiming strategy by the

567 end of the training session, and later reflected in the lack of implicit reach aftereffects. In

568 contrast, using a strongly predictive cue of target workspace separation, Schween et al. (2018)

569 nicely demonstrated significant implicit reach aftereffects in their group that received verbal

570 instructions about how to counter their blocked $60^{\circ} \mathrm{CW}$ and $\mathrm{CCW}$ rotations (Schween et al.,

5712018 ) that were otherwise shown to interfere. Indeed, we found a similar effect whereby dual

572 adaptation as cued by separated target sets were able to elicit reach aftereffects that decreased in

573 size as a function of the distance between workspaces (Baldeo et al., 2013). Critically, we show 
574 here that moderately predictive cues can indeed elicit implicit learning, but in the case of the

575 Static Visual Cue and Instruction Experiments, an ineffective cue even in conjunction with

576 aiming strategies will be unsuccessful.

577 Finally, it should be emphasized that our instructed participants are by proxy provided

578 some explicit component of learning (i.e. through the aiming strategy), while those uninstructed

579 groups (e.g. in Schween et al., (2018)) were able to derive it from visuomotor learning alone. In

580 fact, their participants had relatively decent estimates of the rotations, estimating over half of the

581 rotations' magnitudes in their explicit judgment task, while our explicit reach aftereffects in the

582 Instruction Experiment only reached about a third of the size of our rotations. In light of these

583 key differences, these findings bring up critical points that (1) explicit strategies can support dual

584 learning but only to a limited extent, (2) potentially, that the mechanisms behind dual adaptation

585 differ for smaller and larger rotations, and (3) the possible influence of rotation presentation

586 schedules (pseudo-randomized trial-by-trial vs. alternating blocks of 8 trials per rotation).

587 Altogether our findings show that while intrinsic cues provide an avenue for the motor

588 system to learn two or more visuomotor maps at the same time, it is single-handedly the

589 underlying explicit mechanism that drives learning when the cues are insufficient. This has

590 significant implications in short-term rehabilitation where time is constrained and the motor

591 system must rely on explicit processes to re-learn multiple motor skills, so future studies should

592 investigate how to enhance this explicit process so that learning can be retained over extended

593 periods of time. 
595 Ayala, M. N., ‘t Hart, B. M., \& Henriques, D. Y. P. (2015). Concurrent adaptation to opposing 596 visuomotor rotations by varying hand and body postures. Experimental Brain Research, 233(12), 3433-3445. https://doi.org/10.1007/s00221-015-4411-9

Ayala, M. N., \& Henriques, D. Y. P. (2018). Context-dependent concurrent adaptation to static and moving targets. PLOS ONE. https://doi.org/10.1371/journal.pone.0192476

Baldeo, R., \& Henriques, D. (2013). Dual adaptation to opposing visuomotor rotations with similar hand movement trajectories. Exp Brain Res, 227(2), 231-241. Retrieved from http://www.ncbi.nlm.nih.gov/entrez/query.fcgi?cmd=Retrieve\&db=PubMed\&dopt=Citation

Balitsky Thompson, A. K., \& Henriques, D. Y. (2010). Visuomotor adaptation and intermanual transfer under different viewing conditions. Exp Brain Res, 202(3), 543-552. Retrieved

Brashers-Krug, T., Shadmehr, R., \& Bizzi, E. (1996). Consolidation in human motor memory.

611 Caithness, G., Osu, R., Bays, P., Chase, H., Klassen, J., Kawato, M., ... Flanagan, J. R. (2004). Failure to consolidate the consolidation theory of learning for sensorimotor adaptation tasks. J Neurosci, 24(40), 8662-8671. https://doi.org/10.1523/JNEUROSCI.2214-04.2004

614 Dionne, J. K., \& Henriques, D. Y. (2008). Interpreting ambiguous visual information in motor learning. $J$ Vis, 8(15), 2 1-10. Retrieved from http://www.ncbi.nlm.nih.gov/entrez/query.fcgi?cmd=Retrieve\&db=PubMed\&dopt=Citation 
\&list_uids=19146286

618 Donchin, O., Francis, J. T., \& Shadmehr, R. (2003). Quantifying generalization from trial-by619 trial behavior of adaptive systems that learn with basis functions: theory and experiments in 620 human motor control. J Neurosci, 23(27), 9032-9045. Retrieved from 621 http://www.ncbi.nlm.nih.gov/entrez/query.fcgi?cmd=Retrieve\&db=PubMed\&dopt=Citation $622 \quad \&$ \&ist_uids $=14534237$

623 Galea, J. M., \& Miall, R. C. (2006). Concurrent adaptation to opposing visual displacements 624 during an alternating movement. Exp Brain Res, 175(4), 676-688. Retrieved from http://www.ncbi.nlm.nih.gov/entrez/query.fcgi?cmd=Retrieve \&db=PubMed\&dopt=Citation \&list_uids $=16835793$

Gupta, R., \& Ashe, J. (2007). Lack of adaptation to random conflicting force fields of variable magnitude. J Neurophysiol, 97(1), 738-745. Retrieved from http://www.ncbi.nlm.nih.gov/entrez/query.fcgi?cmd=Retrieve\&db=PubMed\&dopt=Citation \&list_uids=17093124

Heuer, H., \& Hegele, M. (2008). Adaptation to Visuomotor Rotations in Younger and Older Adults. Psychology and Aging. https://doi.org/10.1037/0882-7974.23.1.190

Hinder, M. R., Woolley, D. G., Tresilian, J. R., Riek, S., \& Carson, R. G. (2008). The efficacy of colour cues in facilitating adaptation to opposing visuomotor rotations. Exp Brain Res, 191(2), 143-155. Retrieved from http://www.ncbi.nlm.nih.gov/entrez/query.fcgi?cmd=Retrieve\&db=PubMed\&dopt=Citation \&list_uids=18679663

Howard, I. S., Ingram, J. N., Franklin, D. W., \& Wolpert, D. M. (2012). Gone in 0.6 seconds: the encoding of motor memories depends on recent sensorimotor states. The Journal of 
Neuroscience: The Official Journal of the Society for Neuroscience, 32(37), 12756-12768. https://doi.org/10.1523/JNEUROSCI.5909-11.2012

642 Howard, I. S., Wolpert, D. M., \& Franklin, D. W. (2013). The effect of contextual cues on the 643 encoding of motor memories. Journal of Neurophysiology, 109(10), 2632-2644. https://doi.org/10.1152/jn.00773.2012

645 Howard, I. S., Wolpert, D. M., \& Franklin, D. W. (2015). The value of the follow-through 646

Karniel, A., \& Mussa-Ivaldi, F. A. (2002). Does the motor control system use multiple models and context switching to cope with a variable environment? Exp Brain Res, 143(4), 520-

Krakauer, J. W., Ghilardi, M. F., \& Ghez, C. (1999). Independent learning of internal models for

Osu, R., Hirai, S., Yoshioka, T., \& Kawato, M. (2004). Random presentation enables subjects to adapt to two opposing forces on the hand. Nat Neurosci, 7(2), 111-112. Retrieved from http://www.ncbi.nlm.nih.gov/entrez/query.fcgi?cmd=Retrieve \&db=PubMed\&dopt=Citation \&list_uids $=14745452$

Schween, R., Taylor, J. A., \& Hegele, M. (2018). Plan-based generalization shapes local implicit adaptation to opposing visuomotor transformations. Journal of Neurophysiology. https://doi.org/10.1152/jn.00451.2018 
663 Taylor, J. A., Krakauer, J. W., \& Ivry, R. B. (2014). Explicit and Implicit Contributions to

664 Learning in a Sensorimotor Adaptation Task. Journal of Neuroscience, 34(8), 3023-3032.

665 https://doi.org/10.1523/JNEUROSCI.3619-13.2014

666 Taylor, Jordan A., Klemfuss, N. M., \& Ivry, R. B. (2010). An explicit strategy prevails when the 667 cerebellum fails to compute movement errors. Cerebellum, 9, 580-586.

668 https://doi.org/10.1007/s12311-010-0201-X

669 Wang, L., \& Musseler, J. (2014). Concurrent adaptation to opposite visual distortions:

$670 \quad$ impairment and cue. Psychol Res, 78(4), 453-464. https://doi.org/10.1007/s00426-013-

$671 \quad 0500-1$

672 Werner, S., Van Aken, B. C., Hulst, T., Frens, M. A., Van Der Geest, J. N., Strüder, H. K., \&

673 Donchin, O. (2015). Awareness of sensorimotor adaptation to visual rotations of different

674 size. PLoS ONE, 10(4). https://doi.org/10.1371/journal.pone.0123321

675 Woolley, D. G., de Rugy, A., Carson, R. G., \& Riek, S. (2011). Visual target separation

676 determines the extent of generalisation between opposing visuomotor rotations. Exp Brain

677 Res, 212(2), 213-224. Retrieved from

678 http://www.ncbi.nlm.nih.gov/entrez/query.fcgi?cmd=Retrieve $\& \mathrm{db}=\mathrm{PubMed} \& d o p t=C i t a t i o n$

$679 \quad$ \&list_uids $=21562858$

680 Woolley, D. G., Tresilian, J. R., Carson, R. G., \& Riek, S. (2007). Dual adaptation to two

681 opposing visuomotor rotations when each is associated with different regions of workspace.

682 Exp Brain Res, 179(2), 155-165. Retrieved from

683 http://www.ncbi.nlm.nih.gov/entrez/query.fcgi?cmd=Retrieve\&db=PubMed\&dopt=Citation

$684 \quad$ \&list_uids $=17119942$ 